\title{
Graphite oxidation and SEM as a tool for microstructural investigation
}

\author{
H. Badenhorst* ${ }^{* 1}$ \\ ${ }^{1}$ Department of Chemical Engineering, University of Pretoria, Pretoria, 0002, South Africa
}

The microstructure of graphite and its oxidation have been individually investigated at length. When any catalytic impurities are removed from graphite, oxidative attack takes place preferentially from the exposed edges, or active sites of graphite crystallites. This provides valuable insights regarding the underlying crystallography and fundamental structure of graphite which are not immediately evident from a raw topographical examination using, for example, microscopy. Using new techniques, such as field emission guns and sensitive detectors, modern scanning electron microscopes are capable of operating down to a few hundred volts. When surface features and effects are being considered this is absolutely critical as the low voltages imply low sample penetration of the electrons, thus the true surface morphology of the material can be resolved. In combination, these methods provide a valuable technique for investigating the microstructures found in graphite. The approach has been applied to natural but is even more relevant to synthetic graphite and therefore nuclear materials, which have an exceedingly complex microstructure. The revealed structural qualities can be related to the manufacturing processes in order to gain additional insights into the material and uncover potential options for improving the properties.

Keywords: graphite, natural, synthetic, oxidation, SEM

\section{Introduction}

The oxidation of graphite has been studied for a very long time [1,2] but seldom has it been used a tool for exposing and interpreting the microstructure of graphite. The ideal crystal structure of graphite is well-known and extensively studied [3]. Due to its $\mathrm{sp}^{2}$ bond structure, atoms are arranged in a hexagonal, layered structure, as shown in Fig. 1. This also leads to a bond angle of $120^{\circ}$ between all atoms and the distinctive honeycomb structure.

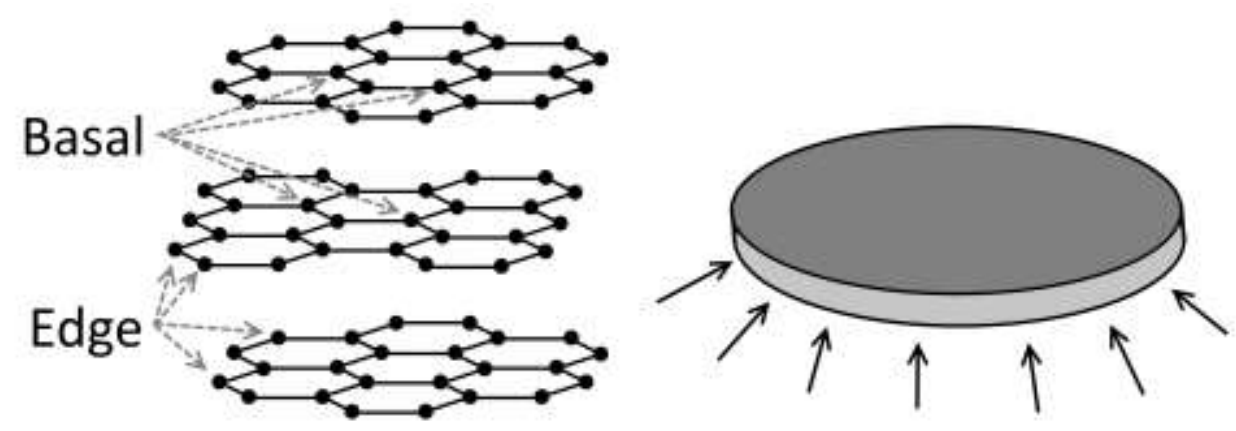

Fig. 1 Graphite crystal structure and primary oxidation direction.

Basal atoms, located at the centre of such a single layer, are extremely tightly and covalently bonded to their neighbours. This makes them very resistant to oxidative attack, except at exceptionally high temperatures. The edge atoms on the other hand can either contain very reactive dangling bonds due to unsatisfied $\mathrm{sp}^{2}$ orbitals or bonds to hetero atoms such as oxygen [4]. These so-called surface complexes are more susceptible to attack and can be thermally desorbed easily. Graphite most commonly occurs naturally in a flake form due to its layered nature. These flakes usually have large aspect ratios being much thinner than they are wide. For these reasons the simplest model for the oxidation of graphite is that of a shrinking disc, reacting only from its edges depicted in Fig. 1. This reaction model can be analytically described to determine the reaction rate as a function of oxidative progression and is found to be a power relationship of 0.5 [5]. However, this behaviour is rarely observed in practice, with graphite exhibiting a very wide range of kinetic responses. The main reasons for these deviations from the idealized behaviour are the presence of impurities and microstructural variations [6].

The objective of this paper is to demonstrate how the underlying microstructure of different graphite materials can be revealed through suitable pre-treatments and the use of high resolution scanning electron microscopy. Oxidative attack is used to remove extraneous features and expose the fundamental crystallinity of the material. These obscuring structures are the result of the material origin or processing steps which are not directly related to the formation mechanism, such as mineral impurity inclusions and beneficiation through milling. The crystallinity of natural and synthetic graphite materials have been evaluated using a variety of bulk characterization techniques ranging from 
Raman spectroscopy to X-ray diffraction. Broadly speaking the materials exhibit very similar characteristics, yet they have very different properties and practical applications. In the manner described, these two, seemingly closely related materials, can be compared based on their true microstructure. This in turn can lead to clear conclusions regarding the relationship between the formation mechanism and the crystallinity of these materials, which is not possible using the as-received raw materials. This in turn can be used to improve manufacturing techniques or for the selection of the correct material for a specific application.

\section{The influence of impurities}

If the catalytic impurities are removed from graphite, oxidative attack is indeed only possible from the exposed edges of the graphite crystallites. However, if this is not done, even trace amounts of impurities on the order of one part per million can drastically disrupt the oxidative progression of the sample edge. Catalytically active impurities can either lead to pit formation or channelling in the graphite [7], of which the latter is visible in Fig. 2(a). These contaminants can also have an extreme impact on the measured reaction rate. The "unzipping" action of these catalysts, through channel formation, leads to a dramatic increase in the number of exposed edges where oxidation can occur. Thus as the catalyst moves around, it is continually creating new reaction zones, increasing the overall rate. Even impurities which are not catalytically active can influence edge development and progression. As oxidation proceeds, these inactive impurities will accumulate at the edges as the flake shrinks. If positioned correctly, these particles can shield the edge from oxidation, thereby creating an inhibition zone. In some cases, a small particle can shield a small region which in turn protects a larger region and so on and so forth. In this manner pyramidal edge structures are created as typified by the formations seen in Fig. 2(b).

a)
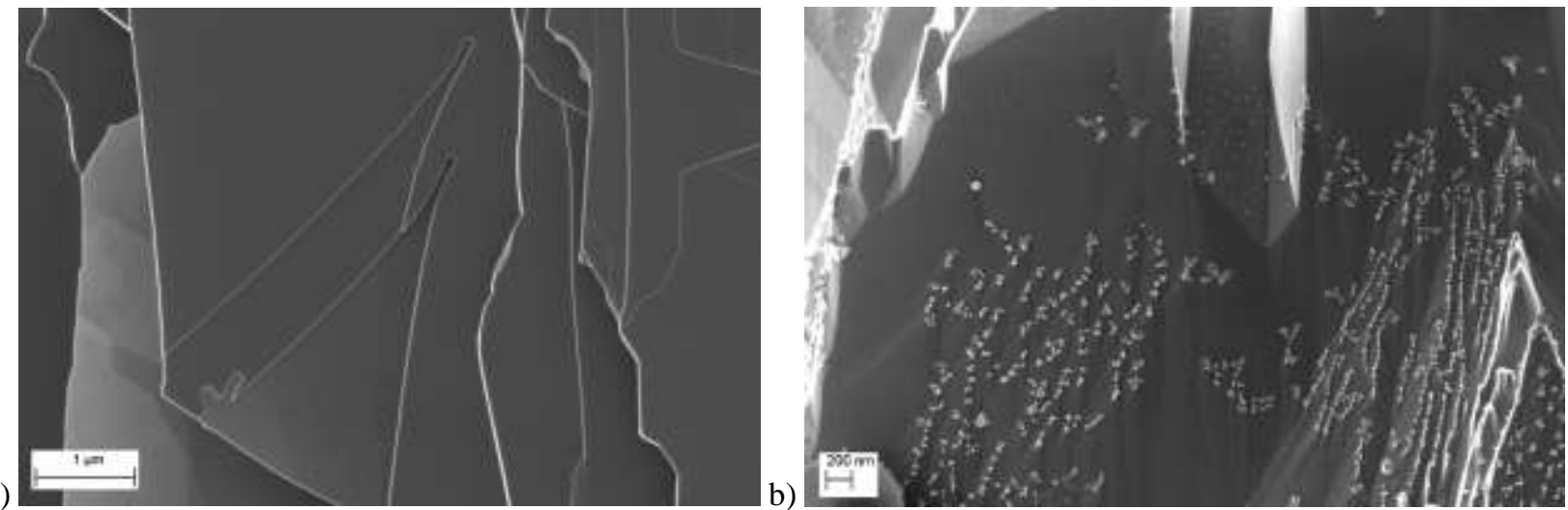

Fig. 2 (a) Impurity channelling in graphite; (b) Impurity accumulation at graphite edges

Depending on mobility and oxidative progression, these particles can also accumulate in coordinated lines, as visible along the ridges in Fig. 2(b). The advancements in modern scanning electron microscopy, such as the use of in-lens detectors and field emission guns are crucial. Without these technologies which enable operation at acceleration voltages of a few hundred volts surface resolution of these particles would not be possible. It is essential when considering the microstructure to discern between effects caused by contaminants and the results of the underlying crystalline structure.

\section{Sample preparation}

Impurity removal may be achieved by acid wash and alkali roasting, however, this may lead to attack and structural damage of the particles, therefore ultra-high temperature $\left(>2500{ }^{\circ} \mathrm{C}\right)$ thermal treatment under inert conditions is preferred. Nonetheless, even if the impurities can be removed in a suitable manner natural graphite still does not exhibit the expected graphite microstructure. Such a purified sample is presented in Fig. 3. 

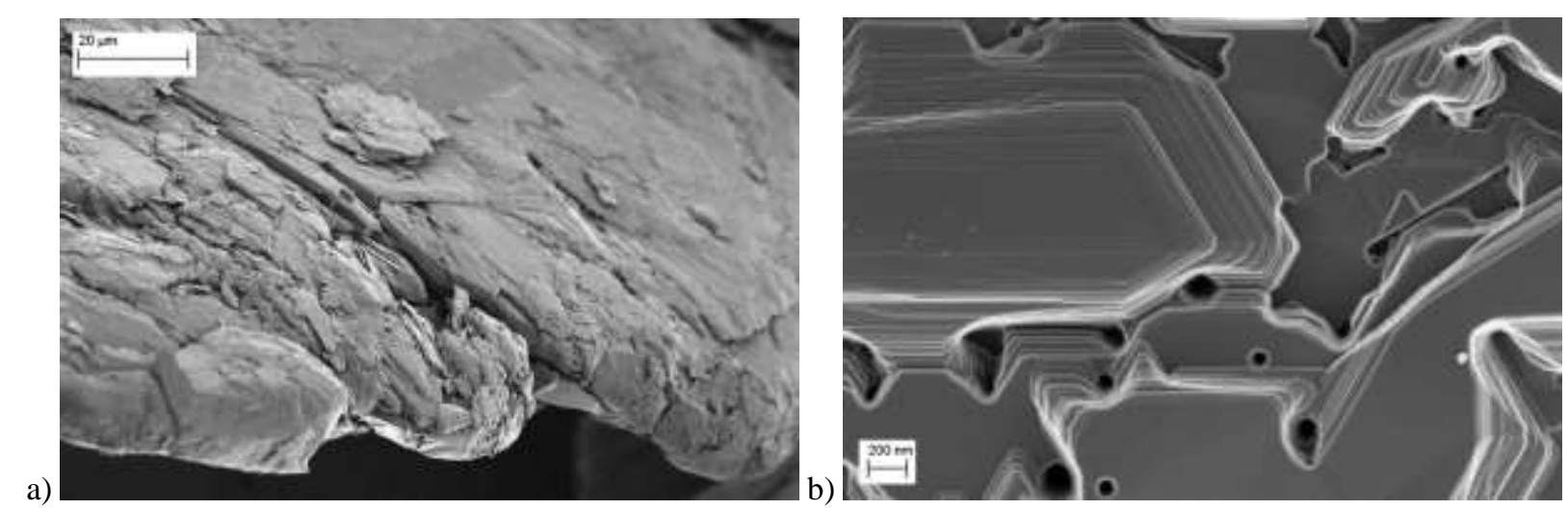

Fig. 3 (a) Purified graphite edge; (b) Surface structures of thermally treated graphite

The flake edges visible in Fig. 3(a) are not flat and exhibit a characteristic curved or bent shape. These formations are caused by the beneficiation process which involves milling of the raw material [8] to liberate the graphite particles from their constituent ores, mostly silicate based minerals. This damage is unavoidable due to the soft nature of the material. It should be noted that even a mild sonication can cause similar structural degradation. Another consequence of the preparation technique is demonstrated in Fig. 3(b). The layered structure of the graphite is clearly visible from this figure along with distinctive polygonal edge arrangements and depressions. These structures are a result of the formation mechanism of the graphite. In this case natural flake graphite is formed by fluid precipitation from high temperature, molten magma during mineralization. Invariably, inclusions of these constituent minerals are trapped within the graphite crystal structure. Upon heating to ultra-high temperatures these impurities can be evaporated from the graphite leaving behind said characteristic formations. Under normal SEM examination of as-received material these impurity regions would be indistinguishable from graphite based on topology but can be identified by in-situ compositional analysis such as energy dispersive X-ray analysis.

These steps constitute physical preparation of the sample itself before analysis. For SEM investigation of graphite samples, very little additional sample preparation is needed. Particles are lightly deposited on adhesive carbon tape which is attached to a flat metal support, usually an aluminium plate. Care should, however, be taken to ensure all particles present on the plate are firmly adhered with the tape. Any loose particles may be dislodged during examination, leading to potential damage to the SEM instrument and possibly destruction of the electron gun filament. For these reasons the sample is extensively sprayed with compressed air to firmly attach the powders to the adhesive and liberate any rogue particles. Additionally, if any volatiles are present, they can be evaporated by a short drying period in a suitable oven. The reason for such limited sample preparation is the high electrical conductivity of the graphite. The graphite freely allows electrons to move through the sample which in turn leads to minimal charging. Charging adversely affects the imaging capability of the SEM and can lead to sample degradation. If the sample is electrically insulating, the sample must be coated to allow for electron dissipation. This is most commonly achieved by sputter coating, either with carbon or gold. In most cases, a single sputter coating will be adequate and is sufficiently thin enough to not disrupt the observed surface features.

\section{Natural graphite microstructure}

In order to elucidate the true underlying microstructure of natural graphite, however, the samples require an oxidative treatment. The extent of this treatment depends on the required information. To remove all extraneous damage caused for example by milling, the samples are oxidized in air or oxygen up to a mass loss of around $30 \%$. At this point all jagged or damaged edge and surface structures will have been reacted away. Due to the nature of the oxidative attack, features with high surface area are removed first, leaving behind the overall, underlying flake structure. This can be seen for the purified, oxidized $\left(800^{\circ} \mathrm{C}\right.$ in pure oxygen), natural graphite flake as shown in Fig. 4(a). 

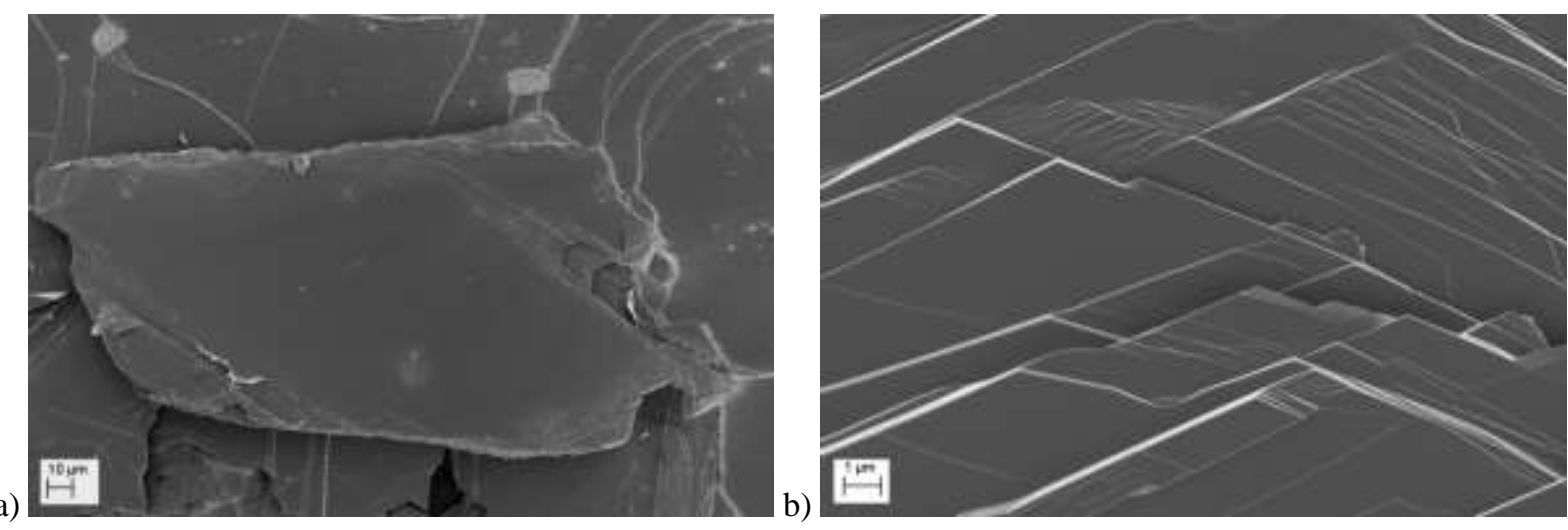

Fig. 4 (a) Oxidized natural graphite flake; (b) Distinctive $120^{\circ}$ edge angles

While not perfect, the roughly rectangular (in this case) flake shape is clearly visible. The basal surface is largely unaffected by the oxidation. Closer inspection confirms the lack of surface attack in this region, consistent with the expected ideal behaviour. The majority of the remaining irregularity is visible only at the exposed edges of the flake where oxidation is occurring. For the flake under consideration it is already clear that not all flakes are disc-shaped. Coincidentally though, there is no expected difference in the oxidation behaviour of a disc compared to a square or rectangle. These regularly shaped flakes are, however, found to be the exception. Most flakes are highly irregular but with a clear polygonal shape, comprised of innumerable combinations of 120,60 and $30^{\circ}$ angles. These variations are caused not only by the unpredictable formation mechanism of the flakes, i.e. precipitation, but also by the underlying crystal structure. The distinctive $120^{\circ}$ angle resulting from the underlying atomic arrangement can clearly be seen in some cases, as in Fig. 4(b), confirming the expected crystal structure. However, as can be deduced from Fig. 1, these edges can recombine with edges moving along different crystallographic directions. This results in the possibility of forming any edge angle that is a multiple of $30^{\circ}$. In this way, a myriad of polygonal structures can be created despite the single, $120^{\circ}$ bond angle.

Some surface irregularities can, however, be found even in the basal surfaces. While these do not readily oxidize, they are clearly visible as kinks or dents in the surface. In most cases these are caused by irregular layering within the flake itself. Due to the growth mechanisms the flake is usually not generated as a single crystal. Depending on conditions, flakes can either be precipitated laterally or growth can be initiated in a spiral fashion, leading to a screw type crystal defect. Multiple stacks, each consisting of a very large number of aligned layers, can be independently grown as part of a single flake. When such a structure is stressed, the stacks can collapse in empty regions where growth was uneven. This results in multi-level folding or kinking of the structure depending on the underlying stack configuration. It has also been observed that a single stack can bend up, over an underlying stack and back down, returning to a level close to its original position. This is made possible by a defect very common in graphite called twinning. As can be seen from Fig. (1) bonds align in certain directions, most notably the armchair direction. Bond rotation along a common axis requires very little energy and can easily occur. Due to this mechanism the basal surface can easily be deformed to accommodate underlying defects.

In general, two possibilities exist for the stacking of individual layers within the graphite crystal. These are $\mathrm{ABAB}$ and $\mathrm{ABCABC}$, which denotes the fact that for the first type every second atomic layer is identical and for the second, every third. These are commonly referred to as hexagonal and rhombohedral stacking. The latter can simply be considered as a stacking fault, or basal dislocation, of the former. In fact, it is found that rhombohedral stacking can be induced in hexagonal graphite through deformation, such as milling. Furthermore, rhombohedral stacking reverts to hexagonal upon heating to intermediate temperatures. Nonetheless, either possibility only results in a slight misalignment of at most three atomic layers with each other. Such a small discrepancy would not lead to an effect that would be visible even on the nanometre resolution scale possible with advanced SEMs. Yet a very wide variety of edge structures can be found in even the most pristine and highly crystalline natural graphite samples. A selection of these edge features are shown in Fig. 5.

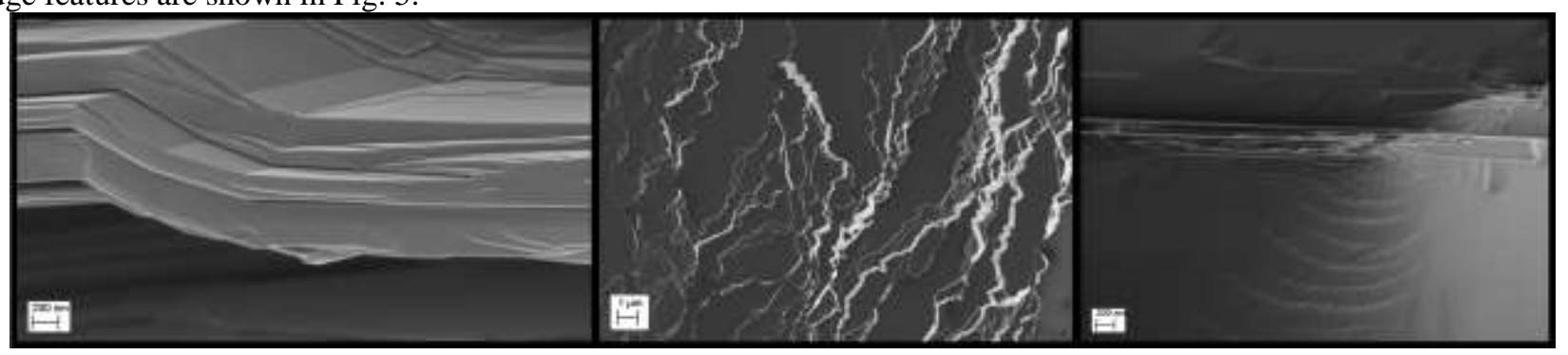

Fig. 5 Natural graphite edge structures 
Certain edge irregularities can be explained in light of the previously discussed crystal defects and others, such as basal edge dislocations. Twinning is usually observed as twin banding, i.e. rotation around a single bond axis followed shortly after by a reverse rotation around the same axis, restoring the original plane orientation. The rotation angle is also thought to be characteristic of a certain material. However, in graphite multiple, successive bandings in the same direction can be observed with widely varying rotational angles. Basal edge dislocations, implying a lateral shift of the basal edge, can account for the observed layering effect, as multiple stacks may be dislocated relative to each other. This can lead to different oxidation behaviour across what appears to be a single crystal but is instead an assembly of misaligned stacks. These dislocations may also account for the gradual appearance and disappearance of edge structures. If the dislocated edge is sufficiently small, the surrounding crystal may collapse around it, effectively restoring it to a close to ideal state. Other observed behaviours are more difficult to explain based on the idealized crystal structure. For example, the appearance of multiple steps across a single edge face which was oxidized a short distance away as one continuous structure, implying a regular near perfect crystal. As pointed out previously, the appearance of polygonal edges comprised of multiple combinations of 30, 60 and $120^{\circ}$ angles can be justified based on considerations of the crystallographic directions. However, in some instances very erratic or even gradually curved edge structures are observed. These can be present in flakes which at other edges exhibit the expected $120^{\circ}$ angles. In situations where two edges are seen to move away from each other as they are oxidized, very anomalous behaviour can be observed at the intersection between these edges. Surfaces which appear to be continuous single crystal structures, can exhibit oxidation across the entire face with curvature inconsistent in any way the basal surface. The reasons for these anomalies remain a mystery and further work is needed to understand the underlying atomic and microstructural arrangements which lead to these features.

The required extent of burn-off varies from sample to sample depending on origin and to a certain extent even from flake to flake within a single sample. This depends heavily on the microstructural information required from the investigation and the microstructures found in the sample. In many cases it is advantageous to prepare a set of oxidized samples, with a gradual progression in the extent of mass loss. This is especially true for kinetic modelling, where the explanation for a rate change during a specific conversion period is usually needed. In general, the temperature at which oxidation is conducted may be irrelevant. However, in some cases, especially where competing reactions take place the structural development which occurs may be strongly influenced. For example, it is well-established that graphite has variable edge reactivity depending on whether a zig-zag or armchair edge is exposed. At temperatures below $900{ }^{\circ} \mathrm{C}$ the reaction rate along zig-zag edges is expected to outweigh reactivity along armchair edges. From an overall flake perspective this may not significantly influence the observed behaviour, however, since twinning bands only occur parallel to armchair edges hexagonal etch pit development within a flake will change orientation substantially. In addition, it should be noted that the underlying assumption thus far has been that oxidation is kinetically limited. If oxidation temperatures reach excessively high values, the reaction may transition into a diffusion dominated scheme. This could lead to uneven oxidation and incorrect conclusions regarding the microstructure. The latter also depends heavily on the choice of oxidation platform. For this investigation a thermo-gravimetric analyser was employed, but in general any oven with sufficient gas circulation could be used. The design of the reaction vessel is however crucial to ensure even gas distribution and homogenous oxidation of the entire sample.

\section{Synthetic graphite microstructure}

While the microstructures found in natural graphite demonstrate some unexpected behaviours, the overall structure still conforms to idealized expectations. The material is comprised of large flat sheets which can only be oxidized at the exposed edge regions. For synthetic graphite the situation is quite different, the complexity and variation of the structures found in these materials is exceptionally extensive. This is despite the fact that traditional bulk characterization methods like X-ray diffraction and Raman spectroscopy reports similar values for the material structural properties [9]. The microstructure of synthetic graphite is still not well understood and it is a topic of ongoing exploration. The topic is of particular interest in the nuclear industry where graphite is extensively used in next generation reactors both as a neutron moderator and structural component. In this case the situation is further complicated by irradiation effects which act to modify the crystal structure. To understand some of the causal factors in the observed complexity it is necessary to appreciate the production process of synthetic graphite.

Unlike natural graphite which is formed by geological processes under extreme conditions, synthetic graphite is produced from a variety of carbonaceous precursors through a few industrial processes. The two primary precursors may be broadly classified as petroleum pitch and coal tar pitch. Petroleum pitch is the heavy residue remaining after the distillation of petroleum products, including decant oil. Coal tar is the residue obtained from coal pyrolysis to produce coke. This material is distilled to yield a heavy product called coal tar pitch. Both materials contain poly-aromatic hydrocarbons with very high aromaticity and carbon to hydrogen ratios. In general, both pitches are solids at room temperature with coal tar pitch having a slightly higher softening range at around $150{ }^{\circ} \mathrm{C}$ compared to the $90{ }^{\circ} \mathrm{C}$ for petroleum pitch. Because of this distinction, each material is used at a different point in the manufacturing process. 
Petroleum pitch is generally used to make the bulk component of the final graphite in the form of needle coke. The pitch is rapidly heated to around $500{ }^{\circ} \mathrm{C}$ and injected into a coking drum. The pitch promptly transforms into a solid, in the process volatile components are liberated creating gas pockets. Due to the injection mode of operation, the bubbles tend to expand and stretch the forming solid in the injection direction. This stretching effect aligns the aromatic molecules in the material and gives the coke its characteristic needle-like texture. The coke is then cut out of the drum and calcined at $1500{ }^{\circ} \mathrm{C}$ for 2 hours to remove any hetero atoms, leaving only pure carbon.

The needle coke is then milled to the desired size and mixed with coal tar pitch. The pitch acts as a binder, linking together the particles during extrusion. This part is then carbonized at $1000{ }^{\circ} \mathrm{C}$ for 2 hours and if required a second pitch impregnation is performed to increase the material density. Finally, the article is graphitized by resistively heating it to $3000{ }^{\circ} \mathrm{C}$ for several hours and allowing it to cool down slowly. The entire process can take several months and artefacts ranging in size from a few $\mathrm{cm}$ to cylinders of several meters with half a meter diameter can be produced. For the purpose of this investigation a small segment of such a synthetic graphite product was crushed and ground into a fine powder. To a certain extent, this procedure breaks the graphite back down into its constituent parts. The graphite particles considered in this investigation are obtained from milled nuclear graphite provided by SGL Carbon (NBG 18 grade). The exact origin of the petroleum and coal tar pitch used in production are unknown. The elongated, thin particles of needle coke are readily discernable in the sample, as can be seen from Fig. 6 .

a)

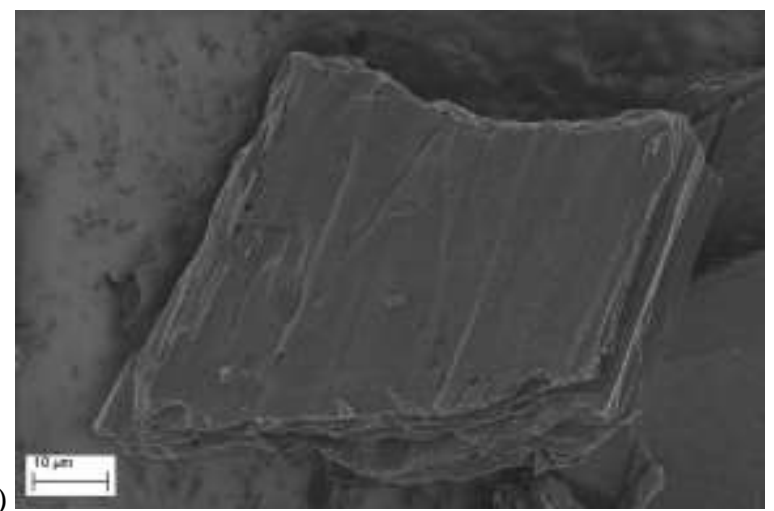

b)

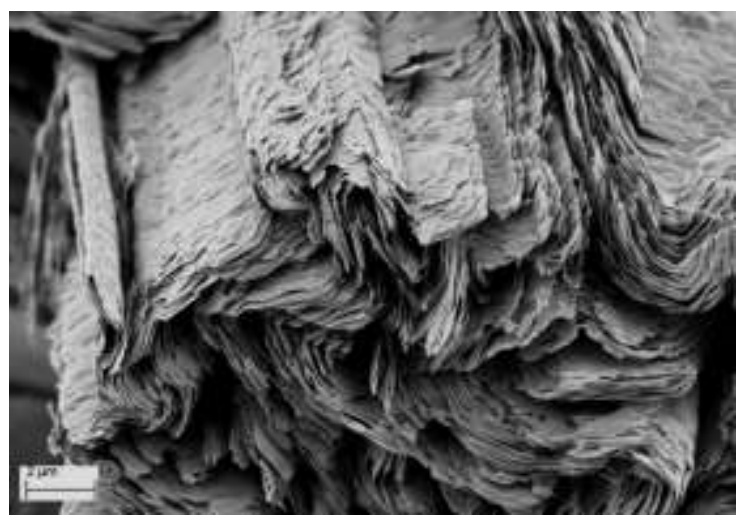

Fig. 6 (a) Synthetic graphite needle coke particle from petroleum pitch; (b) Needle coke particle oblique view

Due to the orientation of very large aromatic molecules along the direction of stretching, the graphite basal plane can be discerned as having formed along this axis. At first glance the particle may appear similar to the rectangular natural graphite flake shown earlier. However, several key differences exist, most notably the creased or folded basal surface. In contrast to the natural graphite which, for the most part, exhibited a very smooth and flat surface, the synthetic material exhibits a somewhat uneven texture with multiple lines or corrugations across the length. The reason for this behaviour can be understood when a needle coke particle is viewed edge on, as in Fig. 6(b). The microstructure is in fact completely different, natural graphite can be thought of as a neat stack of flat sheets of paper. The synthetic graphite structure on the other hand is akin to taking several stacks of a small number of sheets and folding each stack randomly along its long axis many times. These folded stacks are then randomly combined and intertwined to give a single particle. From a distance the flat surfaces of the individual stacks give the impression of an overall flat surface but they are in fact quite uneven and even distinct. At the edge the layering expected of a graphitic structure is quite clear, but there is evidently no single orientation direction. Small stacks of sheets are aligned with each other but they have a random orientation relative to others and the particle as a whole. The observed structure is distinctive and is commonly found in so-called discotic nematic liquid crystals. As fluids containing large, flat crystallites are forced to flow in one direction, the inevitable result is referred to as flow disclinations. These are regions of fluid movement where particles temporarily align and there localised orientation is related to each other and the direction of movement. The fairly well-structured arrangement of needle coke particles is in stark contrast to the utterly chaotic nature of particles derived from the coal tar pitch binder as demonstrated in Fig 7(a). 

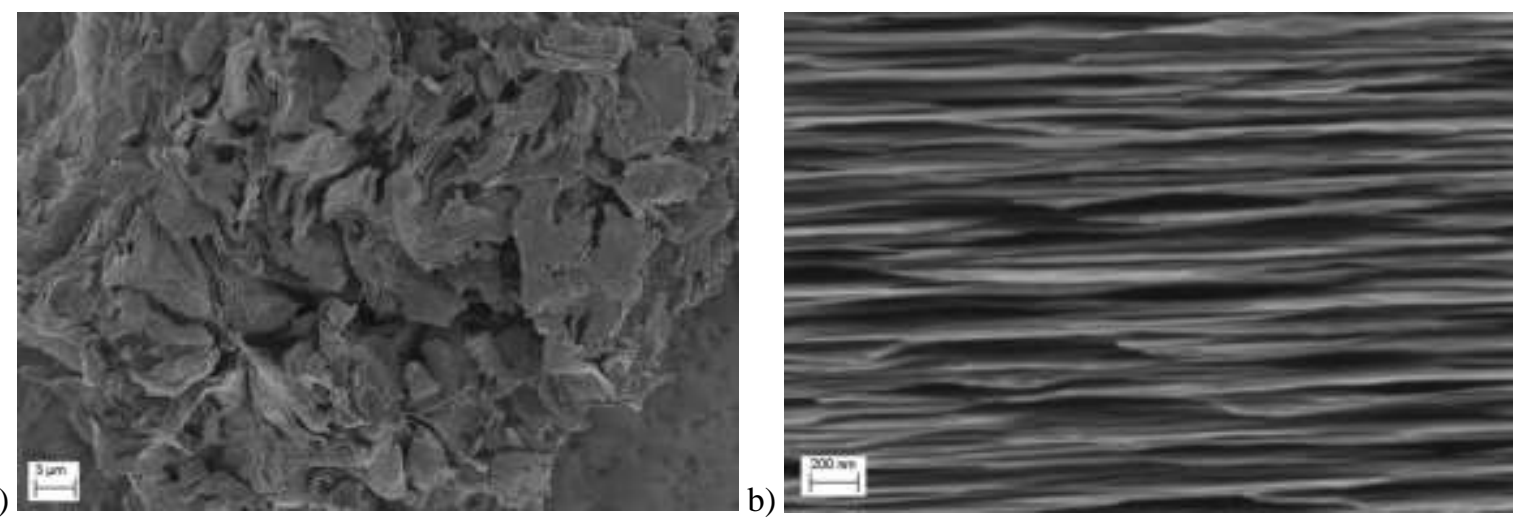

Fig. 7 (a) Synthetic graphite coal tar pitch particle; (b) Needle coke edge structure

Due to the rapid transformation and forces acting on the petroleum pitch at the time of needle coke formation, these flow structures are captured and fixed within the coke microstructure. On the other hand, the coal tar pitch is used as a binder which is then comparatively slowly and progressively heated up to the point of transformation into a solid. Due to this process the pitch is in a relaxed molten state during the conversion into coke and eventually graphite. This results in a very disordered and practically isotropic final microstructure. Despite the disorder when the particle is examined, closely layered regions are still visible. These layered regions have very similar characteristic dimensions for needle coke and pitch, with platelets on the order of less than $100 \mathrm{~nm}$ thick, as seen in Fig. 7(b). This is very dissimilar from the same edge structures visible in natural graphite where continuous, smooth edge sections can be several tens of $\mu \mathrm{m}$ thick. In natural graphite these can be explained in terms of basal edge dislocations, i.e. small lateral shifts of graphitic stacks relative to each other. On the other hand, for synthetic graphite it is difficult to imagine the forces which resulted in such an excessively high number of relative slip planes across a given edge, which in total can also measure up to a $\mu \mathrm{m}$ in overall thickness. Instead this points toward a more fundamental crystallinity difference in the two materials.

This significant disparity in crystallinity is also visible when the basal surface of a needle coke particle is examined more closely. For natural graphite the surface is very smooth, with no visible oxidative attack and the exception of a few kinks or dimples in the surface due to gaps in the underlying structure. Synthetic graphite, on the other hand, exhibits a totally different behaviour as is visible in Fig. 8(a). The basal surface is heavily pockmarked with oxidation occurring virtually everywhere on the surface. This implies that the material is comprised of much smaller crystallites with attack being possible at the grain boundaries where these crystals join together. Thus while the overall structure does exhibit long range order and demonstrates clearly the orientation which was created during the stretching of the liquid precursor through formation, on a fundamental level these structures are made up of tiny crystallites. Judging from the SEM images, individual graphite crystallites are no more than a few tens of nm in width and height.

a)
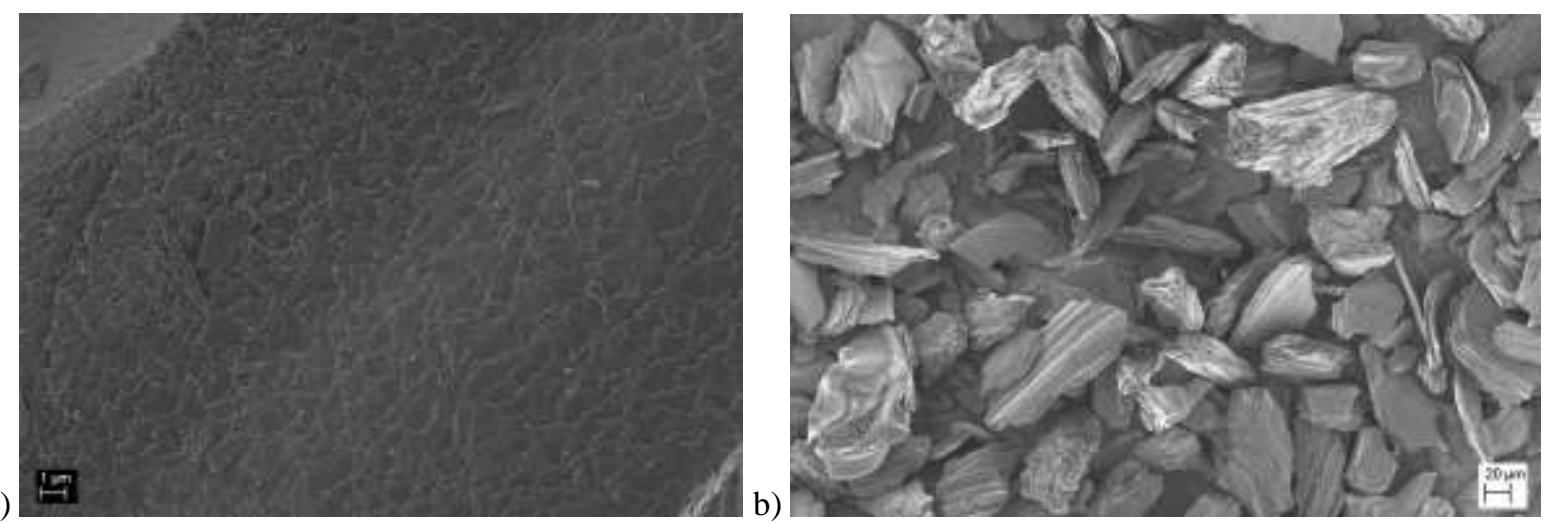

Fig. 8 (a) Synthetic graphite basal surface; (b) Synthetic graphite particle variation

Based on the vast differences in formation mechanism, it is perhaps unsurprising to find such large disparities in crystallinity. Natural graphite is slowly precipitated from a molten liquid whereas synthetic graphite is formed from its poly-aromatic hydrocarbon precursor. Both petroleum and coal tar pitches are known to undergo an intermediate transformation to form graphite, namely the formation of mesophase. Mesophase is a liquid crystal state where polycondensation has led to the creation of very large aromatic molecules. Size estimates through time of flight mass spectrometry place these molecules in the 1500 AMU range. These are the building blocks which eventually form the graphite crystal stacks. While this may sound large on the atomic scale, on a length scale it does not amount to much. If aromatic rings were simply linked in a chain, a single ring wide, a pure carbon molecule of 1500 AMU would measure 
less than $10 \mathrm{~nm}$ in size. This is, however, close to the length scale on which oxidative attack is visible in the synthetic graphite. Additional transformations are known to occur in the solid state once the material has transformed into a coke. So it is likely that further linkages of these molecules occur up to a point. The edges of these large molecules then form the grain boundaries of the eventual graphite crystallites.

An additional fact which should be considered is the planar nature of graphite. It is unlikely that the molecules formed by the polycondensation of smaller aromatic molecules are perfectly flat. This would imply that the layering resulting from the stacking of these molecules would be curved. It is possible that this leads to strain in the matrix as the material is graphitized and heated to $3000{ }^{\circ} \mathrm{C}$. In turn this would result in some molecular rearrangement but this would be limited to the breaking and reforming of bonds in the most stressed regions, rather than the free migration of single carbon atoms. Hence the extent to which these defects can be corrected is limited. This in combination with the liquid phase formation mechanism leads to a tremendous variation in the possible particle shapes present in synthetic graphite, as demonstrated in Fig. 8(b). While many particles have been fractured along the expected thin, elongated lines anticipated for needle coke, a multitude of completely random particles are also visible. These are the result of flow regions where several disclinations intersect, in a three dimensional sense. Such particles are in sharp contrast to the expected flat graphitic shapes expected from the ideal graphite crystal structure. Nonetheless, these particles are in fact highly graphitic with a well-defined crystal structure as demonstrated by the X-ray diffraction analysis. While the layering can be uncovered by examining these formations closely, on the whole they present a completely irregular and random microstructure.

\section{Conclusions}

The deductions regarding the differences and similarities between natural and synthetic graphite would not have been possible without the combination of oxidation and high surface resolution SEM. The true behaviours of the as-received materials are obscured due to contaminants and structural damage caused by processing. Only by purifying and gradually exposing the underlying crystal structure can the microstructural features be understood in context of the material formation mechanism. The surface features relevant to these insights cannot be resolved at high acceleration voltages due to excessive surface penetration. Thus without recent advancements in scanning electron microscopy such progress would not have been possible. On the other hand, the resolution which has been achieved has illuminated certain aspects of the behaviour which cannot readily be explained given our current understanding of the ideal graphite crystal. This leaves the door open for future investigations to fully elucidate the underlying crystalline microstructure and its structural characteristics developed through oxidation.

Acknowledgements This research is supported by the South African Research Chairs Initiative of the Department of Science and Technology and National Research Foundation of South Africa (Grant No 97994). Any opinion, finding and conclusion or recommendation expressed in this material is that of the author(s) and the NRF does not accept any liability in this regard..

\section{References}

[1] Gulbransen EA, Andrew KF. Reactions of artificial graphite: Surface oxide formation and surface roughness studies in relation to oxidation of artificial graphite at temperatures of $25^{\circ} \mathrm{C}$ and between 425 to $575{ }^{\circ} \mathrm{C}$. Industrial and Engineering Chemistry. 1952; 44:1039-1044.

[2] Walker PL. Carbon - an old but new material. American Scientist. 1962; 50:259-293.

[3] Pierson HO. Handbook of Carbon, Graphite, Diamond and Fullerenes. Properties, Processing and Applications. Noyes Publications, New Jersey, US; 1992.

[4] Radovic LR. The mechanism of $\mathrm{CO} 2$ chemisorption on zigzag carbon active sites: A computational chemistry study. Carbon. 2005; 43:907-915.

[5] Badenhorst H, Rand B, Focke W. A generalized solid state kinetic expression for reaction interface-controlled reactivity. Thermochimica Acta. 2013; 562:1-10.

[6] Badenhorst H, Focker WW. Geometric effects control isothermal oxidation of graphite flakes. Journal of thermal analysis and calorimetry. 2012; 108:1141-1150.

[7] Badenhorst H. Novel simulation technique for the prediction of complex oxidation behaviour in natural graphite flakes. Chemical Engineering Science. 2013; 104:117-124.

[8] Badenhorst H, Focke W. Comparative analysis of graphite oxidation behaviour based on microstructure. Journal of Nuclear Materials. 2013; 442:75-82.

[9] Badenhorst H. Microstructure of natural graphite flakes revealed by oxidation: limitations of XRD and Raman techniques for crystallinity estimates. Carbon. 2014; 66:674-690. 